\title{
Data-Based Model Refinement Using Retrospective Cost Optimization
}

\author{
Anthony M. D'Amato,* \\ University of Michigan, Ann Arbor, Michigan, 48109 , United States of America \\ Sunil. L. Kukreja, ${ }^{\dagger}$ \\ NASA Dryden Flight Research Center, Edwards, California, 93523, United States of America \\ Dennis S. Bernstein. ${ }^{\ddagger}$ \\ University of Michigan, Ann Arbor, Michigan, 48109, United States of America
}

\begin{abstract}
Data-based model refinement improves the fidelity of a model based on empirical observations. The model refinement structure is a closed-loop system consisting of an initial model, which is assumed to be known, and a feedback correction, which is unknown. We take advantage of the similarity between the feedback structure of model refinement and adaptive feedback control by replacing the unknown correction term with a retrospectivecost-based adaptive control law. The adaptive control law thus estimates the unknown subsystem. We investigate scenarios in which the initial model is derived from physical principles, such as mass-spring-damper systems, in order to obtain estimates of stiffness and damping coefficients. Furthermore, we identify dynamic subsystems for a linear initial model and a nonlinear initial model.
\end{abstract}

\section{Introduction}

This paper focuses on the problem of using data to improve model fidelity. We are motivated by the scenario in which an initial model is derived from idealized physical principles. Although these models can provide good numerical resolution in space and time, they may inherit errors from numerous sources. For example, such models are often based on simplifying approximations and may be subject to discretization errors that cause numerical viscosity and violation of divergence-free constraints. Additional model errors may result from model reduction techniques, which are required to attain a manageable model size for applications such as feedback control.

Modeling errors may be irrelevant for some applications, such as conceptual tradeoff studies, but can adversely affect applications such as data assimilation (for example, ensemble Kalman filtering) and feedback control. Using data to improve an initial model can thus increase the value of an initial model. The potential applications of this technology encompass virtually all disciplines that rely on high-dimensional models, for example, structural, fluid, thermal, kinetic, and electromagnetic models with applications to vehicles and devices as well as natural systems, such as the atmosphere and space environments. In all of these applications, data-based model refinement can rectify modeling errors arising from idealized assumptions, numerical effects, and model simplification.

Models serve a variety of purposes by capturing physical processes at varying levels of resolution. A high-resolution model is desirable when the goal is to understand scientific phenomena, whereas a coarser model may be preferable when the goal is to capture essential details in an efficient manner. Consequently, the fidelity of a model must be gauged against its intended usage.

Most models are constructed from collections of interconnected sub-models, which in turn are based on both physical laws and empirical observations. For example, the core of a model might be the Navier-Stokes

\footnotetext{
*NASA GSRP Fellow, Department of Aerospace Engineering, 1320 Beal, and AIAA student member.

$\dagger$ Engineer, DFRC-RC, and AIAA member.

¥Professor, Department of Aerospace Engineering, 1320 Beal Avenue, and AIAA member.
} 
or MHD equations, while chemistry, heating, and friction terms may be modeled using either first principles submodels or empirical relations that may have different levels of fidelity and complexity. Physical laws embody first-principles knowledge, whereas empirical observations may include relations that are based on the statistical analysis of data. Physics can provide the backbone of a model, while empirical (data-based) relations can flesh out details that are beyond the ability of the physical model (for example, sub-grid-scale phenomena).

In data-based model refinement we assume the availability of an initial model, which may incorporate both physical laws and empirical observations. The components of the initial model may have varying degrees of fidelity, reflecting knowledge of the relevant physics as well as the availability of data. With this initial model as a starting point, our goal is to use additional measurements to refine the model. This problem is known as model correction, empirical correction, model refinement, or model updating; relevant literature includes finite-element modeling, ${ }^{1-4}$ meteorology ${ }^{5-7}$ feedback control, ${ }^{8,9}$ applications to health monitoring, ${ }^{13}$ and algorithms. ${ }^{10-12}$ This literature is surprisingly sparse given the potential benefits of using data to improve models.

We consider the case in which the initial model includes components that have some uncertainty. For example, the physics of a subsystem may not be well understood, and alternative subsystem models may be hypothesized. The uncertain physics of a subsystem can range from the simplest case of an unknown parameter (such as a rate constant), to a multivariable spatially dependent static mapping (such as a conductivity tensor or boundary conditions), to a fully dynamic relationship among multiple variables (such as reaction kinetics). The difficulty of identifying these phenomena from empirical data depends on something we call accessibility, which refers to the degree of separation between the data and the unknown subsystem.

The highest degree of accessibility arises when two variables are measured and the unknown subsystem is a static mapping between the variables. In this case, linear or nonlinear regression can provide a parametric or nonparametric estimate of the unknown mapping. Multivariable extensions of this situation are more challenging, but ultimately are tractable due to the high degree of accessibility.

In this paper we consider a technique for subsystem identification when the degree of accessibility is low. In this situation, limitations on the availability of measured data prevent the use of linear or nonlinear regression techniques. Although brute-force nonconvex optimization methods can be used to estimate unknown maps, we use the available measurements in a more sophisticated way to estimate the unknown subsystem.

Model refinement is concerned with the identification of a specified subsystem of a larger overall model. The principal challenge of model refinement is the situation in which the subsystem of interest has low accessibility, that is, when neither the inputs nor the outputs of the subsystem are measured. As noted in, ${ }^{10-13}$ this problem is equivalent to a problem of adaptive control theory.

Figure 1 shows a block diagram of recursive model refinement. Each block and each signal is labeled to denote its uncertainty status. The blocks labeled "Known Subsystem" and "Unknown Subsystem" represent the physical system, whose inputs include known and unknown inputs. These subsystems are connected through feedback, which captures how each subsystem impacts the other. Serial and parallel interconnections can also be considered, but feedback interconnection provides the greatest generality in practice. The majority of the system dynamics are assumed to be included in the "Known Subsystem" block, while the "Unknown Subsystem" block includes static or dynamic maps that are poorly known. The objective is to use data to obtain a model and, if desired, better physical understanding of the "Unknown Subsystem" block.

The lower part of the diagram in Figure 1 constitutes the "Simulated System." The "Physics Model," which is implemented in computation, captures the dynamics of the "Known Subsystem" and serves as the initial model. This model is interconnected by feedback with the block labeled "Identified Physics," which is refined (updated) recursively as data become available. This model refinement occurs through the "Physics Update" procedure as denoted by the diagonal arrow, which is a tuning procedure that recursively identifies the unknown physics to provide a model of the "Unknown Subsystem" block. This tuning procedure is driven by the model-error signal $z$, which is the difference between the measured data from the "Physical System" and the computed output of the "Simulated System."

When cast in the form of a block diagram in Figure 1, it becomes evident that the model refinement problem is deeply related to an adaptive control problem. We thus apply techniques for adaptive control that are sufficiently general and computationally tractable to address the features of high-dimensional physically meaningful applications.

To address the model refinement problem, we apply techniques developed for adaptive control, as described in. ${ }^{22-24}$ These techniques are distinct from standard adaptive control approaches in several crucial 


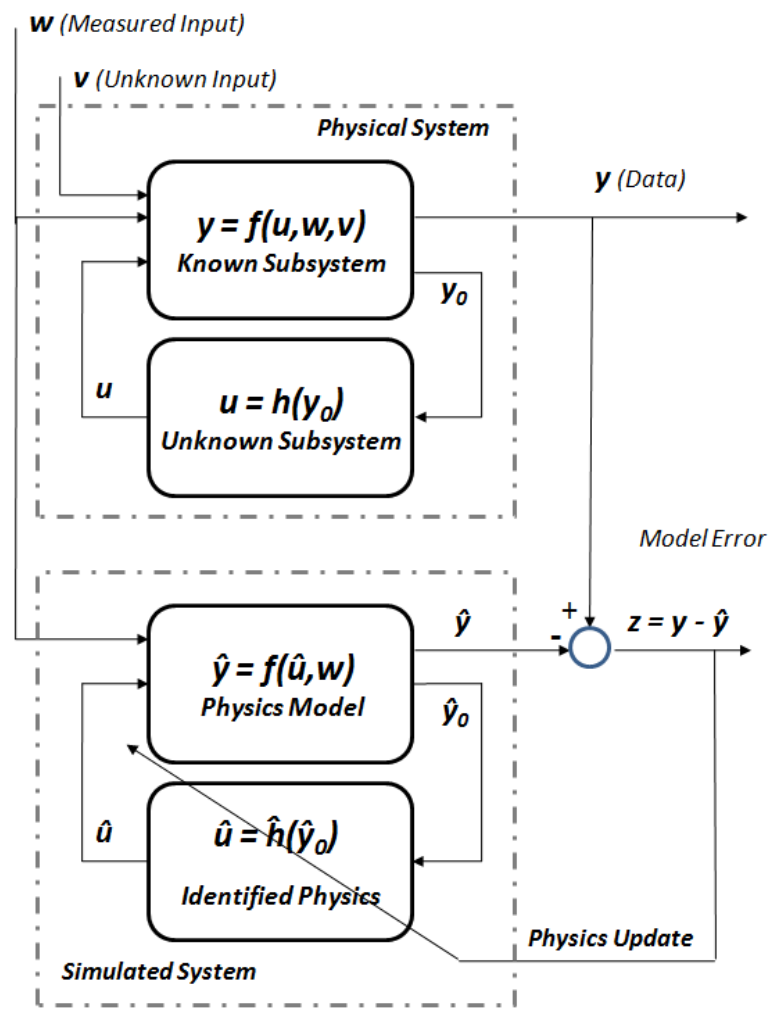

Figure 1. This block diagram illustrates the model refinement problem, where the goal is to identify the "Unknown Subsystem" of the "Physical System." By depicting this problem as a block diagram, it becomes evident that the model refinement problem is equivalent to a problem of adaptive control.

ways. Specifically, the approach of ${ }^{22-24}$ requires minimal modeling information concerning the "Known Subsystem," and is applicable to a wide range of adaptive control problems, including command following, disturbance rejection, stabilization, and model following. This algorithm utilizes a surrogate cost function that entails a closed-form quadratic (and thus convex and tractable) optimization step. Furthermore, the approach of ${ }^{22-24}$ is digital, using discrete-time measured data, and thus the algorithm is well-suited for model refinement. Surprisingly, the controller requires only information about the zeros of the controlled system; no information about the poles is needed. Even more surprisingly, the controller requires only sign information (the sign of the high-frequency gain) and knowledge of the nonminimum-phase zeros of the system. This result shows definitively that nonminimum-phase zeros are the crucial modeling information needed for adaptive control. Basic details of the algorithm and a proof for the minimum-phase case are described in. ${ }^{23-25}$ Algorithms for the nonminimum-phase are presented in. ${ }^{26}$

For model refinement, the problem of interest is a combination of both adaptive command following and adaptive disturbance rejection, where the "command" to be followed is the measured data $y$, and the "disturbance" to be rejected is the unknown input $v$.

The novel feature of the technique developed $\mathrm{in}^{22,23}$ is the use of a retrospective cost criterion to update the estimate of the "Unknown Subsystem." Unlike many adaptive control techniques that are limited to systems with minimum-phase zeros and low relative degree, this approach is effective for systems with arbitrary poles, zeros, and relative degree. This flexibility allows us to apply the technique of retrospective cost adaptive control to the problem of model refinement.

In this paper we apply retrospective-cost-based model refinement to single- and multi-degree-of-freedom systems to investigate fundamental properties, such as accuracy and rate of convergence. In particular, we consider lumped structures in which the damping and/or stiffness are uncertain, and we use either position or rate measurements. We also consider the case in which the structure involves an unknown substructure, 
for which we identify a dynamic model. We demonstrate the technique on an experimental setup involving an RLC circuit with uncertain components. Finally, we consider the case where the initial model is nonlinear, specifically, we apply the refinement method to a space weather modeling problem.

\section{Problem Formulation}

From Figure 1, consider the transfer function representation of the known subsystem $y=f(u, w)$,

$$
\left[\begin{array}{c}
y \\
y_{0}
\end{array}\right]=\left[\begin{array}{cc}
G_{w y} & G_{u y} \\
G_{w y_{0}} & G_{u y_{0}}
\end{array}\right]\left[\begin{array}{l}
w \\
u
\end{array}\right]=G\left[\begin{array}{l}
w \\
u
\end{array}\right],
$$

where $G$ is the known initial model, $y$ is the output data, $w$ is the measured input signal, $y_{0}$ is the output to the unknown subsystem $h$, and $u$ is the output of $h$. Furthermore, $u=h\left(y_{0}\right)$ is represented by the transfer function

$$
\begin{aligned}
u & =G_{\Delta}\left[\begin{array}{l}
y_{0} \\
w
\end{array}\right] \\
& =G_{\Delta, y_{0}} y_{0}+G_{\Delta, w} w
\end{aligned}
$$

We stress that the signals $u$ and $y_{0}$ are not accessible, and thus $G_{\Delta}$ cannot be directly identified. From (1) and (3), we obtain the closed loop transfer function from $w$ to $y$,

$$
y=\left[G_{w y}+G_{u y}\left(G_{\Delta, y_{0}}\left[I-G_{u y_{0}} G_{\Delta, y_{0}}\right]^{-1}\left[G_{w y_{0}}+G_{u y_{0}} G_{\Delta, w}\right]+G_{\Delta, w}\right)\right] w,
$$

The goal is to estimate the unknown subsystem $\hat{G}_{\Delta}$, such that the simulated system

$$
\hat{y}=\left[G_{w y}+G_{u y}\left(\hat{G}_{\Delta, y_{0}}\left[I-G_{u y_{0}} \hat{G}_{\Delta, y_{0}}\right]^{-1}\left[G_{w y_{0}}+G_{u y_{0}} \hat{G}_{\Delta, w}\right]+\hat{G}_{\Delta, w}\right)\right] w,
$$

matches the physical system, that is, $z=y-\hat{y}$ is small.

To identify the feedback term $G_{\Delta}$ using a given initial model $G$, we use an adaptive feedback model structure to identify $\hat{G}_{\Delta}=\left[\hat{G}_{\Delta, y_{0}} \hat{G}_{\Delta, w}\right]$. To achieve model matching, we minimize the performance variable $z$ in the presence of the measured signal $w$. In particular, we use the retrospective cost optimization (RCO) adaptive control algorithm. ${ }^{23}$ The only signals available to the controller are the measurement $y$, the estimated simulated system output $\hat{y}$ and thus the error signal $z$.

\section{Retrospective-Cost Model Identification}

In this section, we review the cumulative retrospective cost adaptive control algorithm developed in. ${ }^{24}$ The algorithm depends on several parameters that are selected a priori. Specifically, $n_{\mathrm{c}}$ is the estimated controller order, and $P(0)$ is the initial error covariance.

Consider a strictly proper time-series controller of order $n_{\mathrm{c}}$, such that the control $u(k)$ is given by

$$
u(k)=\sum_{i=1}^{n_{\mathrm{c}}} M_{i}(k) u(k-i)+\sum_{i=1}^{n_{\mathrm{c}}} N_{i}(k) y_{0}(k-i)+\sum_{i=1}^{n_{\mathrm{c}}} L_{i}(k) w(k-i),
$$

where, for all $i=1, \ldots, n_{\mathrm{c}}, M_{i}: \mathbb{N} \rightarrow \mathbb{R}^{l_{u} \times l_{u}}, N_{i}: \mathbb{N} \rightarrow \mathbb{R}^{l_{u} \times l_{y}}$ and $L_{i}: \mathbb{N} \rightarrow \mathbb{R}^{l_{u} \times l_{w}}$ are determined by the adaptive control law presented below. The control (6) can be expressed as

$$
u(k)=\theta(k) \phi(k),
$$

where

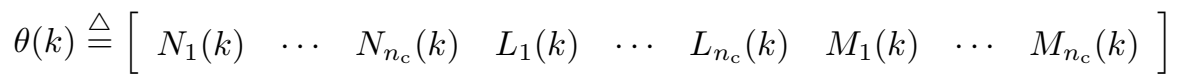


and

$$
\phi(k) \triangleq\left[\begin{array}{c}
y(k-1) \\
\vdots \\
y\left(k-n_{\mathrm{c}}\right) \\
w(k-1) \\
\vdots \\
w\left(k-n_{\mathrm{c}}\right) \\
u(k-1) \\
\vdots \\
u\left(k-n_{\mathrm{c}}\right)
\end{array}\right] \in \mathbb{R}^{n_{\mathrm{c}}\left(l_{u}+l_{y}+l_{w}\right)} .
$$

Next, we represent (1) as the time-series model from $u$ and $w$ to $z$ given by

$$
z(k)=\sum_{i=1}^{n}-\alpha_{i} z(k-i)+\sum_{i=d}^{n} \beta_{i} u(k-i)+\sum_{i=0}^{n} \gamma_{i} w(k-i),
$$

where $\alpha_{1}, \ldots, \alpha_{n} \in \mathbb{R}, \beta_{d}, \ldots, \beta_{n} \in \mathbb{R}^{l_{z} \times l_{u}}, \gamma_{0}, \ldots, \gamma_{n} \in \mathbb{R}^{l_{z} \times l_{w}}$, and the relative degree $d$ is the smallest non-negative integer $i$ such that the $i$ th Markov parameter of $G$, is nonzero.

Next, we define the retrospective performance

$$
\begin{aligned}
\hat{z}(\hat{\theta}, k) \triangleq & \sum_{i=1}^{n}-\alpha_{i} z(k-i)+\sum_{i=d}^{n} \beta_{i} \theta(k-i) \phi(k-i) \\
& +\sum_{i=0}^{n} \gamma_{i} w(k-i) \\
& +\sum_{i=d}^{\nu} \bar{\beta}_{i}[\hat{\theta}-\theta(k-i)] \phi(k-i),
\end{aligned}
$$

where $\nu \geq d, \hat{\theta} \in \mathbb{R}^{l_{u} \times\left(n_{c}\left(l_{y}+l_{u}\right)\right)}$ is an optimization variable used to derive the adaptive law, and $\bar{\beta}_{d}, \ldots, \bar{\beta}_{\nu} \in$ $\mathbb{R}^{l_{z} \times l_{u}}$. The parameters $\nu$ and $\bar{\beta}_{d}, \ldots, \bar{\beta}_{\nu}$ must capture the information included in the first nonzero Markov parameter and the nonminimum-phase zeros from $u$ to $z \cdot{ }^{27}$ In this paper, we let $\bar{\beta}_{d}, \ldots, \bar{\beta}_{\nu}$ be the coefficients of the numerator polynomial matrix of the transfer function from $u$ to $z$, that is, $\nu=n$ and, for $i=d, \ldots, n$, $\bar{\beta}_{i} \triangleq \beta_{i}$. For other choices of the parameters $\nu$ and $\bar{\beta}_{d}, \ldots, \bar{\beta}_{\nu}$, see. ${ }^{27}$

Next, it follows from (8), (9), and the selection of $\bar{\beta}_{d}, \ldots, \bar{\beta}_{\nu}$ that

$$
\hat{z}(\hat{\theta}, k)=z(k)+\sum_{i=d}^{n} \beta_{i}[\hat{\theta}-\theta(k-i)] \phi(k-i) .
$$

Defining $\hat{\Theta} \triangleq \operatorname{vec} \hat{\theta} \in \mathbb{R}^{n_{c} l_{u}\left(l_{y}+l_{u}\right)}$ and $\Theta(k) \triangleq \operatorname{vec} \theta(k) \in \mathbb{R}^{n_{c} l_{u}\left(l_{y}+l_{u}\right)}$, it follows that

$$
\begin{aligned}
\hat{z}(\hat{\Theta}, k) & =z(k)+\sum_{i=d}^{n} \Phi_{i}^{\mathrm{T}}(k)[\hat{\Theta}-\Theta(k-i)] \\
& =z(k)-\sum_{i=d}^{n} \Phi_{i}^{\mathrm{T}}(k) \Theta(k-i)+\Psi^{\mathrm{T}}(k) \hat{\Theta},
\end{aligned}
$$

where, for $i=d, \ldots, n$,

$$
\Phi_{i}(k) \triangleq \phi(k-i) \otimes \beta_{i}^{\mathrm{T}} \in \mathbb{R}^{\left(n_{c} l_{u}\left(l_{y}+l_{u}\right)\right) \times l_{z}},
$$

where $\otimes$ represents the Kronecker product, and

$$
\Psi(k) \triangleq \sum_{i=d}^{n} \Phi_{i}(k) .
$$


Now, define the cumulative retrospective cost function

$$
J(\hat{\Theta}, k) \triangleq \frac{1}{2} \sum_{i=0}^{k} \lambda^{k-i} \hat{z}^{\mathrm{T}}(\hat{\Theta}, i) R \hat{z}(\hat{\Theta}, i)+\frac{1}{2}(\Theta(k)-\Theta(0))^{\mathrm{T}} Q(\Theta(k)-\Theta(0)),
$$

where $\lambda \in(0,1]$, and $R \in \mathbb{R}^{l_{z} \times l_{z}}$ and $Q \in \mathbb{R}^{\left(n_{c} l_{u}\left(l_{y}+l_{u}\right)\right) \times\left(n_{c} l_{u}\left(l_{y}+l_{u}\right)\right)}$ are positive definite. Note that $\lambda$ serves as a forgetting factor, which allows more recent data to be weighted more heavily than past data.

The cumulative retrospective cost function (12) is minimized by a recursive least-squares (RLS) algorithm with a forgetting factor. Therefore, $\hat{J}(\hat{\Theta}, k)$ is minimized by the adaptive law

$$
\begin{aligned}
& \Theta(k+1)=\Theta(k)-P(k) \Psi(k) \times\left[\lambda R^{-1}+\Psi^{\mathrm{T}}(k) P(k) \Psi(k)\right]^{-1} z_{\mathrm{R}}(k), \\
& P(k+1)=\frac{1}{\lambda} P(k)-\frac{1}{\lambda} P(k) \Psi(k) \times\left[\lambda R^{-1}+\Psi^{\mathrm{T}}(k) P(k) \Psi(k)\right]^{-1} \Psi^{\mathrm{T}}(k) P(k),
\end{aligned}
$$

where $P(0)=Q^{-1}, \Theta(0) \in \mathbb{R}^{n_{c} l_{u}\left(l_{y}+l_{u}\right)}$, and the retrospective performance measurement $z_{\mathrm{R}}(k) \triangleq \hat{z}(\Theta(k), k)$. Note that the retrospective performance measurement is computable from (11) using measured signals $z, y$, $u, \theta$, and the matrix coefficients $\beta_{d}, \ldots, \beta_{n}$. The cumulative retrospective cost adaptive control law is thus given by (13), (14), and

$$
u(k)=\theta(k) \phi(k)=\operatorname{vec}^{-1}(\Theta(k)) \phi(k) .
$$

The key feature of the adaptive control algorithm is the use of the retrospective performance (11), which modifies the performance variable $z(k)$ based on the difference between the actual past control inputs $u(k-d), \ldots, u(k-n)$ and the recomputed past control inputs $\hat{u}(\hat{\Theta}, k-d) \triangleq \operatorname{vec}^{-1}(\hat{\Theta}) \phi(k-d), \ldots, \hat{u}(\hat{\Theta}, k-$ $n) \triangleq \operatorname{vec}^{-1}(\hat{\Theta}) \phi(k-n)$, assuming that the current controller $\hat{\Theta}$ had been used in the past.

Note that the direct retrospective cost adaptive controller presented in this section requires knowledge of the coefficients $\beta_{d}, \ldots, \beta_{n}$. These coefficients can be extracted from the model of the known subsystem.

\section{Dynamic Feedback Estimation}

To demonstrate the algorithm (13)-(15), we assume we have an initial model that represents the known part of the system. The unknown subsystem that we wish to identify is linear and either static or dynamic.

Consider the mass-spring-damper structure shown in Figure 2 modeled by

$$
m_{1} \ddot{x}+c_{1} \dot{x}+k_{1} x=w,
$$

where $m_{1}, c_{1}, k_{1}$ are the mass, damping, and stiffness, respectively, and $w$ is the force input. As shown in Figure 2, the mass is also connected to an unknown impedance $G_{\Delta}$, which applies force to the mass in response to the velocity of the mass. We obtain the state space representation of the known subsystem

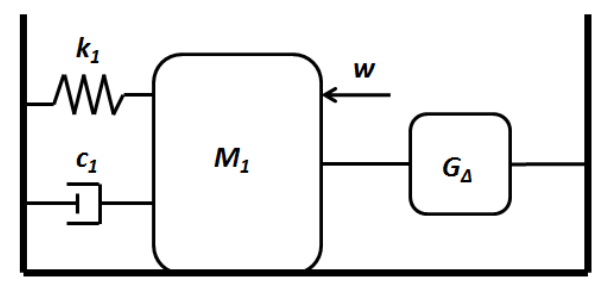

Figure 2. A single degree of freedom mass-spring-damper system connected to an unknown impedance. 


$$
\begin{aligned}
{\left[\begin{array}{l}
\dot{x} \\
\ddot{x}
\end{array}\right] } & =\left[\begin{array}{cc}
0 & 1 \\
-\frac{k_{1}}{m_{1}} & -\frac{c_{1}}{m_{1}}
\end{array}\right]\left[\begin{array}{l}
x \\
\dot{x}
\end{array}\right]+\left[\begin{array}{c}
0 \\
\frac{1}{m_{1}}
\end{array}\right] w, \\
y & =\left[\begin{array}{ll}
0 & 1
\end{array}\right]\left[\begin{array}{l}
x \\
\dot{x}
\end{array}\right],
\end{aligned}
$$

where $x$ and $\dot{x}$ are the position and velocity, respectively, of the mass. Furthermore, $A=\left[\begin{array}{cc}0 & 1 \\ -\frac{k_{1}}{m_{1}} & -\frac{c_{1}}{m_{1}}\end{array}\right]$, $B=\left[\begin{array}{c}0 \\ \frac{1}{m_{1}}\end{array}\right]$, and $C=\left[\begin{array}{ll}0 & 1\end{array}\right]$. Finally, we write the subsystem in transfer function form $G=C(s I-$ $A)^{-1} B$, where the closed loop with the unknown impedance is $G_{\mathrm{cl}}=\frac{G}{1-G G_{\Delta}}$.

We demonstrate the method by choosing $m_{1}=1, k_{1}=30, c_{1}=5$, and $G_{\Delta}=\frac{10}{s+2}$. We choose $n_{c}=5$, which is an overestimate of the order of $G_{\Delta}, P(0)=10^{8} I$, and $\beta$ consists of 10 nonzero Markov parameters of $G$. We note that $G$ and $G_{\Delta}$ are converted to discrete time using a zero-order hold. We retain the same notation for continuous and discrete systems for convenience. The left plot of Figure 3(a) compares frequency
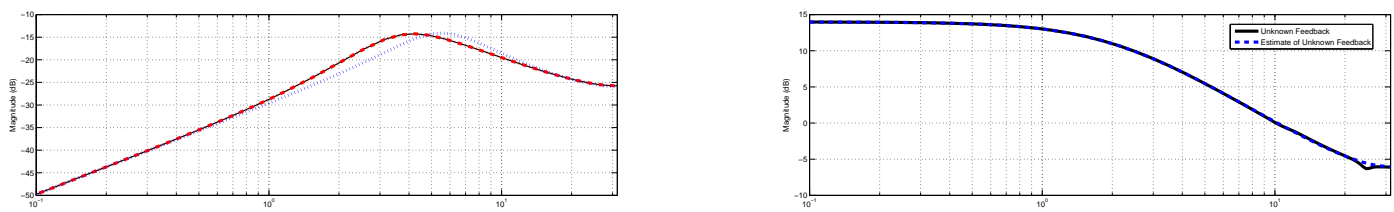

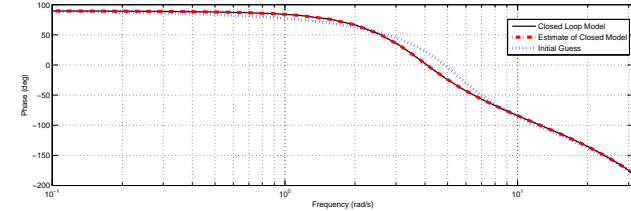

(a)

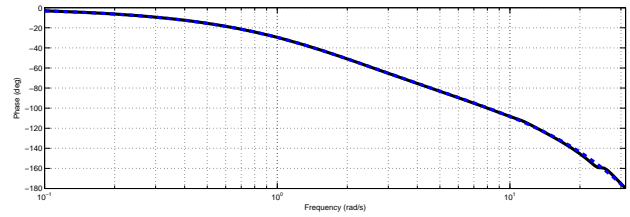

(b)

Figure 3. The left plot is a frequency response comparison of the initial model, the closed loop, and the estimated closed loop using the identified unknown feedback. The right plot is a frequency response comparison of the unknown feedback and the identified feedback.

response of the closed-loop systems. The difference between the initial model and the closed loop is reduced by adding in the identified $\hat{G}_{\Delta}$, where the estimated closed-loop model frequency response is identical to the closed-loop model, the blue dotted line is the initial model with no correction added. Figure 3(b) compares the frequency response of the discrete $G_{\Delta}$ and $\hat{G}_{\Delta}$. The frequency responses are identical.

\section{Static Feedback Estimation}

For systems with a known model structure it is possible to estimate specific unknown parameters. Consequently, estimates of model parameters can be refined using data. To demonstrate this, consider the mass-spring-damper structure shown in Figure 4. The goal is to estimate changes in stiffness and damping using only empirical data, namely, knowledge of the driving force $w$ and output data $y$, specifically, position and/or velocity information.

The equations of motion for this system are

$$
M \ddot{x}+C \dot{x}+K x=F
$$




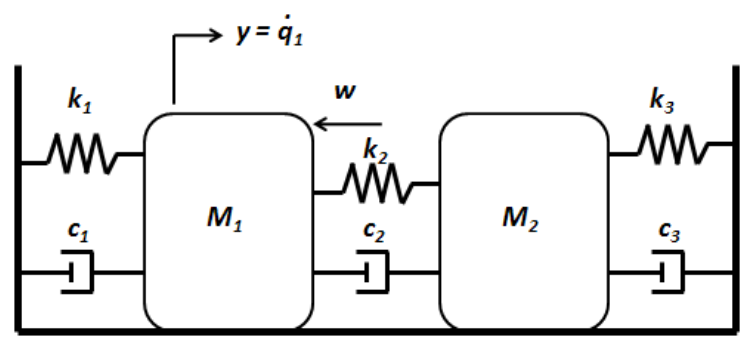

Figure 4. Mass-spring-damper 2-DOF structure.

where

$$
\begin{aligned}
x & =\left[\begin{array}{l}
q_{1} \\
q_{2}
\end{array}\right], M=\left[\begin{array}{cc}
m_{1} & 0 \\
0 & m_{2}
\end{array}\right], C=\left[\begin{array}{cc}
c_{1}+c_{2} & -c_{2} \\
-c_{2} & c_{2}+c_{3}
\end{array}\right], \\
K & =\left[\begin{array}{cc}
k_{1}+k_{2} & -k_{2} \\
-k_{2} & k_{2}+k_{3}
\end{array}\right], \text { and } F=\left[\begin{array}{l}
f_{1} \\
f_{2}
\end{array}\right] .
\end{aligned}
$$

The mass-spring-damper system can be represented in state space form as

$$
A=\left[\begin{array}{cc}
0_{2 \times 2} & I_{2} \\
-M^{-1} K & -M^{-1} C
\end{array}\right], \quad B=\left[\begin{array}{cc}
0_{2 \times 1} & \left(M^{-1} F\right)^{\mathrm{T}}
\end{array}\right]^{\mathrm{T}}, \quad C=\left[\begin{array}{cccc}
0 & 0 & 0 & 1
\end{array}\right], \text { and } D=0 .
$$

We now consider a system where a change in global stiffness and damping has occurred, namely, (20) and (21) become

$$
\ddot{x}=\left[\begin{array}{cc}
0_{2 \times 2} & I_{2} \\
M^{-1}(-K+\Delta K) & M^{-1}(-C+\Delta C)
\end{array}\right] x+\left[\begin{array}{c}
0 \\
M^{-1}
\end{array}\right] w,
$$

where $\Delta K$ and $\Delta C$ are the changes in stiffness and damping, respectively. The changes to the system can then be written in feedback as

$$
\ddot{x}=\left[\begin{array}{cc}
0_{2 \times 2} & I_{2} \\
-M^{-1} K & -M^{-1} C
\end{array}\right]\left[\begin{array}{l}
x \\
\dot{x}
\end{array}\right]+\left[\begin{array}{c}
0 \\
M^{-1}
\end{array}\right] u+\left[\begin{array}{c}
0 \\
M^{-1}
\end{array}\right] w,
$$

where $u=\Delta K x+\Delta C \dot{x}$.

With variations in the model parameters in feedback, it is possible to estimate $\Delta K$ and $\Delta C$ using retrospective cost model refinement. However, the estimate $\hat{G}_{\Delta}$ of the unknown feedback obtained using retrospective cost optimization is a dynamic model, where $\Delta K$ and $\Delta C$ are static. We thus set $n_{c}=1$, and demonstrate numerically that estimates of $\Delta K$ and $\Delta C$ can be obtained by computing the DC gain of $\hat{G}_{\Delta}$. Furthermore, to simulate these systems, the models must be converted from continuous time to discrete time, which modifies the physical representation of the continuous-time models. However, we demonstrate that useful parameter estimates can still be obtained, and the error tends to decrease as the sampling rate increases.

\section{A. Single Degree of Freedom Structure with Uncertain Stiffness}

We estimate a change in stiffness for a single degree of freedom system, where $K=0.6, \Delta K=0.5, C=0.8$, $\Delta C=0$. We note again that (21) is discretized using a zero order hold. The measurement is the velocity of the mass. Figure $5(\mathrm{~b})$ is the frequency response of $\hat{G}_{\Delta}$. The DC gain of this plant is approximately $\Delta K$. In 


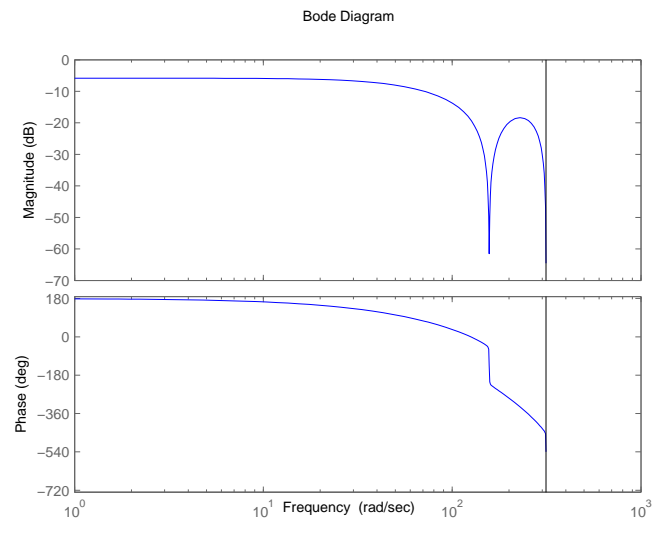

(a)

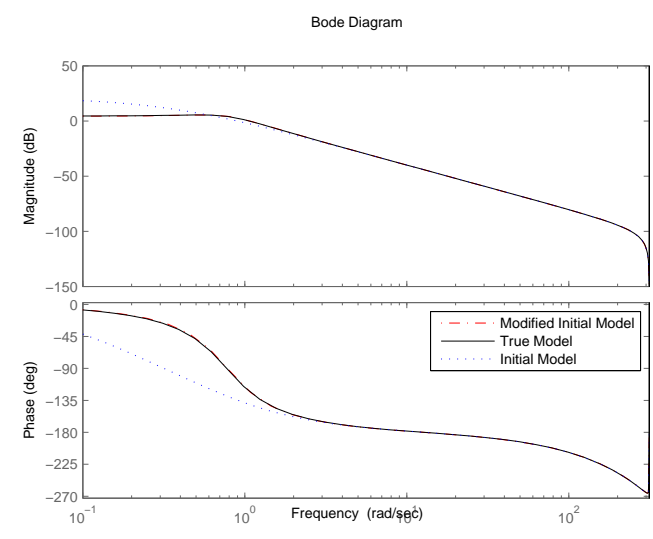

(b)

Figure 5. The left plot is the frequency response of $\hat{G}_{\Delta}$. The DC gain of this plant is approximately $\Delta K$. The right plot is a frequency response comparison of the closed loop models.

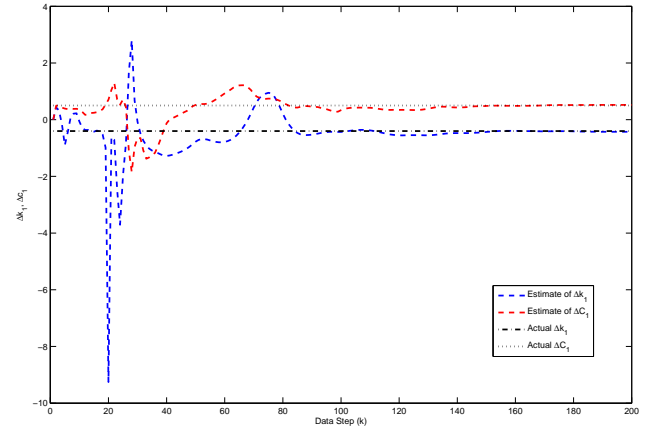

(a)

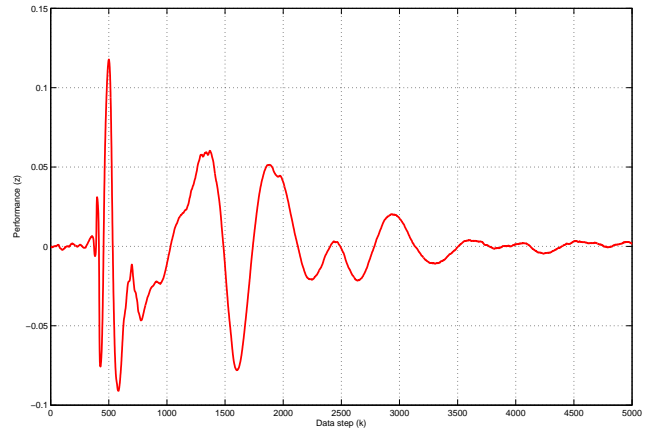

(b)

Figure 6. Single degree of freedom system with unknown changes in stiffness and damping. The left plot is the DC gain of the controller for each time step of the simulation, and the black lines are the actual changes in stiffness and damping. The right plot is the performance variable, which is the difference between the updated model output and the system.

the next example we estimate changes in both stiffness and damping, where $K=0.7, \Delta K=-0.4, C=0.4$, and $\Delta C=0.5$. We choose $n_{c}=1, P(0)=10^{6} I$, and $\beta$ is the first 15 nonzero Markov parameters of the initial model $G$. Figure $6(\mathrm{a})$ shows the parameter estimates, which are the DC gains of the controller as the algorithm converges. Figure $6(\mathrm{~b})$ is the difference between the actual system and the refined model.

\section{B. Two Degree of Freedom}

For the multiple degree of freedom case, we wish to refine our estimate of the global stiffness matrix $\Delta K$, by refining the values of $k_{1}$ and $k_{3}$. For this problem $k_{1}=0.7, \Delta k_{1}=0.4, k_{3}=0.6$, and $\Delta k_{3}=0.5$, the damping coefficients are $c_{1}=c_{2}=c_{3}=0.8$ and $k_{2}=0.5$ is constant. We choose $n_{c}=1, P(0)=10^{3} I$, and $\beta$ is the first 10 nonzero Markov parameters of the initial model $G$. Figure $7\left(\right.$ a) show the estimates of $\Delta k_{1}$ and $\Delta k_{2}$ as a function of data steps. Figure $7(\mathrm{~b})$ show the difference between the actual system and the refined model. 


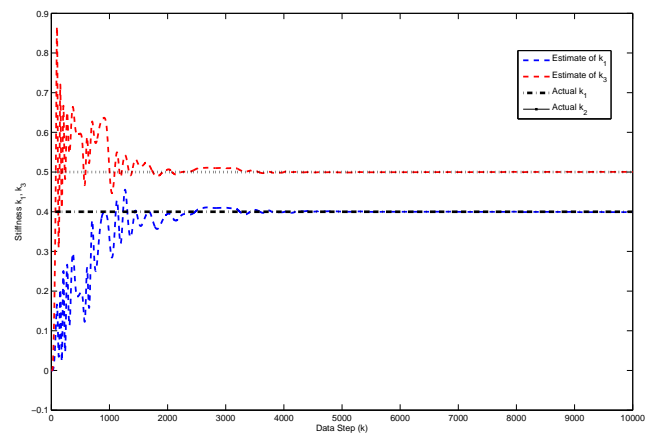

(a)

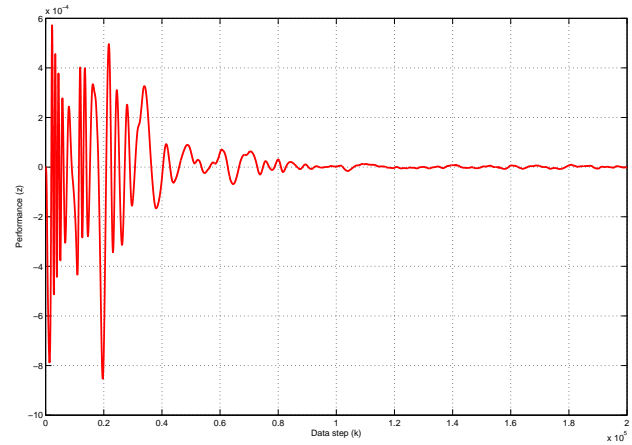

(b)

Figure 7. Two degree of freedom system with unknown changes to $k_{1}$ and $k_{3}$. The left plot is the DC gain of the controller for each time step of the simulation, and the black lines are the actual changes in stiffness and damping. The right plot is the performance variable, which is the difference between the updated model output and the output of the true system.

\section{Experimental Results}

To demonstrate the model refinement method experimentally, we construct a series resistor-inductorcapacitor (RLC) circuit, which is analogous to the mass-spring-damper system. Consider the RLC circuit shown in Figure 8 modeled by

$$
L \ddot{x}+R \dot{x}+\frac{1}{C_{\mathrm{d}}} x=u
$$

where $L, C_{\mathrm{d}}$, and $R$ are the inductor, capacitor, and resistor values, respectively, and $w$ is the input voltage. We obtain the state space representation of the circuit

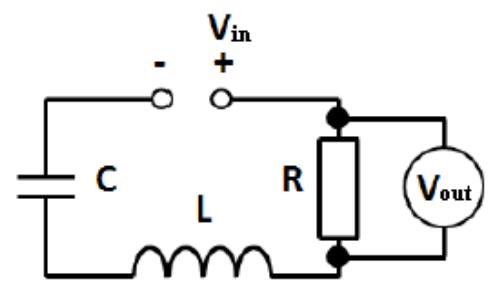

Figure 8. A series resistor-inductor-capacitor (RLC) circuit, where voltage is measured across the resistor.

$$
\begin{aligned}
{\left[\begin{array}{l}
\dot{x} \\
\ddot{x}
\end{array}\right] } & =\left[\begin{array}{cc}
0 & 1 \\
-\frac{1}{L C_{\mathrm{d}}} & -\frac{R}{L}
\end{array}\right]\left[\begin{array}{l}
x \\
\dot{x}
\end{array}\right]+\left[\begin{array}{c}
0 \\
\frac{1}{L}
\end{array}\right] u, \\
y & =\left[\begin{array}{ll}
0 & R
\end{array}\right]\left[\begin{array}{l}
x \\
\dot{x}
\end{array}\right],
\end{aligned}
$$


where $x$ and $\dot{x}$ are the charge and current, respectively, of the circuit. Furthermore, we write the state space equations for a circuit with an unknown change in capacitance $\Delta C_{\mathrm{d}}$ and inductance $\Delta L$ as

$$
\begin{aligned}
{\left[\begin{array}{c}
\dot{x} \\
\ddot{x}
\end{array}\right] } & =\left[\begin{array}{cc}
0 & 1 \\
-\frac{1}{(L+\Delta L)\left(C_{\mathrm{d}}+\Delta C_{\mathrm{d}}\right)} & -\frac{R}{L+\Delta L}
\end{array}\right]\left[\begin{array}{l}
x \\
\dot{x}
\end{array}\right]+\left[\begin{array}{c}
0 \\
\frac{1}{L+\Delta L}
\end{array}\right] w, \\
y & =\left[\begin{array}{ll}
0 & R
\end{array}\right]\left[\begin{array}{l}
x \\
\dot{x}
\end{array}\right],
\end{aligned}
$$

where $u=G_{\Delta_{x}} x+G_{\Delta_{\dot{x}}} \dot{x}+G_{\Delta_{w}} w$. We then compute an estimate $\Delta \hat{C}_{\mathrm{d}}$ of $\Delta C_{\mathrm{d}}$ and an estimate $\Delta \hat{L}$ of $\Delta L$ from the converged adaptive controller by using

$$
\begin{aligned}
\Delta \hat{L} & =\frac{L}{\hat{G}_{\Delta, w}}-L=\frac{-R L}{-R+\hat{G}_{\Delta, \dot{x}}}-L, \\
\Delta \hat{C}_{\mathrm{d}} & =-L\left(\frac{-1}{C_{\mathrm{d}}}+\hat{G}_{\Delta, x}\right)^{-1}(L+\Delta L)^{-1}-C_{\mathrm{d}} .
\end{aligned}
$$

We assemble a circuit with $R=250 \Omega, L+\Delta L=55 \mathrm{mH}$, and $C_{\mathrm{d}}+\Delta C_{\mathrm{d}}=23.5 \mu \mathrm{F}$. We assume that we do not have knowledge of $\Delta C_{\mathrm{d}}$ or $\Delta L$, but we estimate $C_{\mathrm{d}}=1 \mathrm{~F}$ and $L=2 \mu \mathrm{H}$. The model (26) is discretized using a zero order hold. We drive the circuit using zero-mean, Gaussian white noise, and we measure the voltage across the resistor. The driving signal and measurement are recorded using a DSPACE setup.

We implement RCO to obtain estimates of the transfer functions $\hat{G}_{\Delta, x}$ and $\hat{G}_{\Delta, w}$. Figure 9 shows the history of the performance variable $\hat{y}-y$.

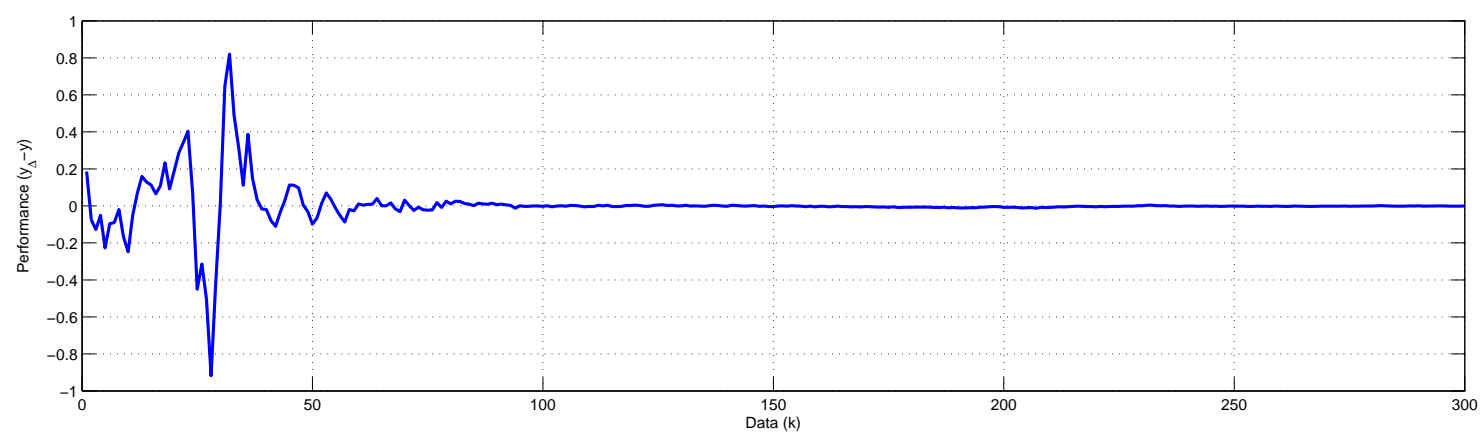

Figure 9. This plot shows the history of the performance variable $\hat{y}-y$.

Figure 10(a) compares the frequency response of the initial model, the actual system, and the refined model, in discrete-time.

\section{Application of Model Refinement to Ionospheric Dynamics Estimation}

To illustrate adaptive model refinement, we consider the problem of estimating dynamic processes in space weather prediction. This problem is challenging due to the fact that we do not assume the availability of measurements that can serve as inputs or outputs to the "Unknown Subsystem" modeling the unknown physics. In other words, the objective of the identification in this particular application is inaccessible relative to the available measurements.

We use the Global Ionosphere Thermosphere Model (GITM) ${ }^{30}$ to simulate the chemistry and fluid dynamics in a 1D column in the ionosphere-thermosphere. The temperature structure of the thermosphere depends on many factors, such as the Sun's intensity in extreme ultraviolet (EUV) wavelengths, eddy diffusion in the lower thermosphere, radiative cooling of the $\mathrm{O}_{2}$ and $\mathrm{NO}$, frictional heating, and the thermal conductivity. 


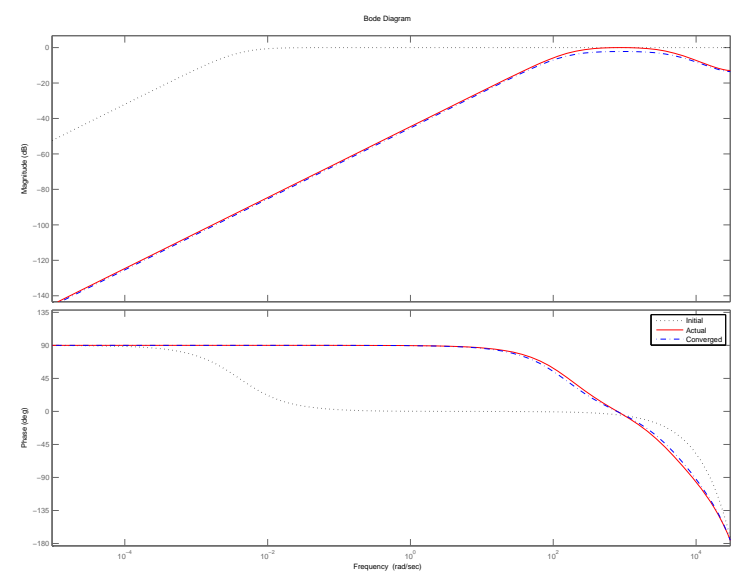

Figure 10. This plot shows the history of the performance variable $\hat{y}-y$.

To illustrate model refinement in the case of an unknown dynamic subsystem, the NO radiative cooling was removed from GITM to provide the initial model but retained in another instance of GITM to provide the truth model. The goal is to reproduce the missing process. This is nontrivial since the functional form and dynamics of the cooling process is assumed to be unknown. We assume only that something is missing from the energy equation, and that it is most likely a function of temperature. The dynamics of the cooling are estimated at three different altitudes, connecting the other altitudes through linear interpolation. The thermospheric density is utilized as data at $407 \mathrm{~km}$ altitude from a simulated truth model that includes NO cooling. The result of the model refinement in Figures 11 and 12 demonstrates that this technique captures the actual dynamics in GITM. The height profile of the cooling matches the actual cooling. Furthermore, the temporal variation of the maximum cooling matches the cooling simulated by the model.

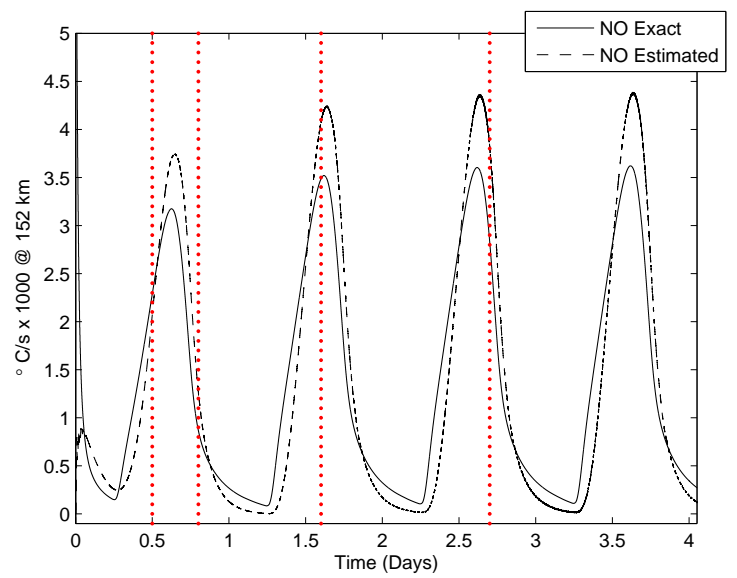

Figure 11. This plot shows the difference between the actual NO cooling included in the truth model and the cooling estimated by the model-refinement technique as a function of time at a specific altitude $(152 \mathrm{~km})$. The vertical dashed lines are the time instances when the altitude vs. NO cooling plots in Figure 12 are taken.

This technique can thus be used to refine and improve an initial model that is either uncertain or erroneous. In turn, the improved model provides a more accurate foundation for data assimilation aimed at wind and density estimates in the presence of solar storm disturbances. Figure 13 compares the model 

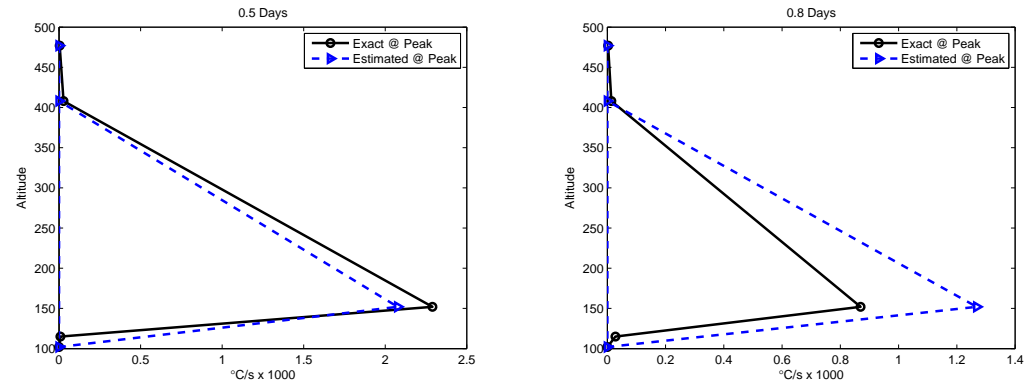

(a) NO cooling as function of altitude at (b) NO cooling as function of altitude at 0.5 days.

0.8 days.
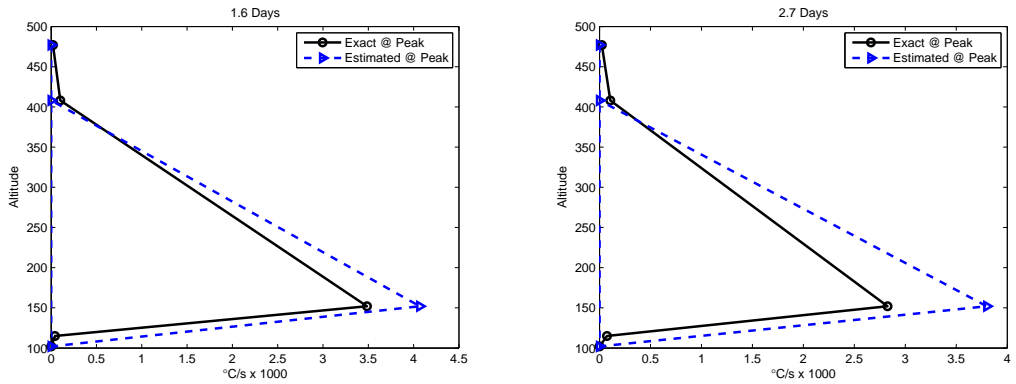

(c) NO cooling as function of altitude at (d) NO cooling as function of altitude at 1.6 days. 2.7 days.

Figure 12. These plots show the difference between the actual NO cooling included in the truth model and the cooling estimated by the model-refinement technique as a function of altitude at a given time. Cooling is along the horizontal axis, while altitude is along the vertical axis. The blue dashed line is the estimated value. The measured data are taken at an altitude of $407 \mathrm{~km}$. The vertical dashed lines in Figure 11 are the time instances when the altitude vs. NO cooling plots are taken.

without correction versus the model with correction, both of which are baselined against the truth model. Without data-based model refinement, the estimated density measurements degrade as time increases.

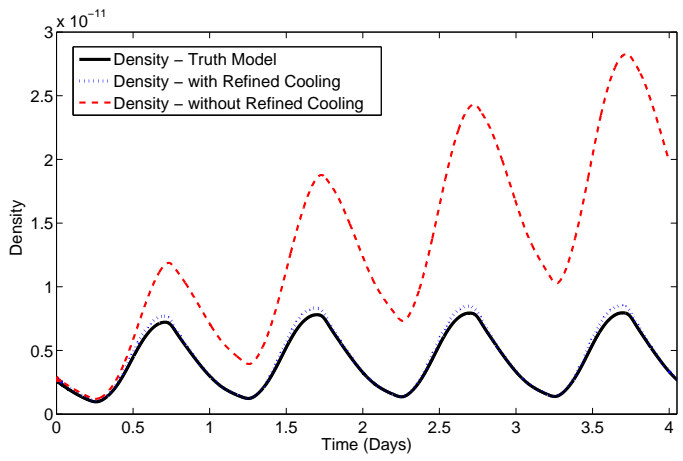

Figure 13. This plot shows the difference between the density measurements for the initial model, where no correction is made, and the model with the refined subsystem versus the truth model. We note that with model refinement, the refined model is able to track the truth model, whereas, in the case that no correction is made, the density measurements degrade as time increases. 


\section{Conclusion}

In this paper we applied a cumulative retrospective cost optimization adaptive control algorithm to the problem of model refinement. The model refinement structure is a closed-loop system consisting of an initial model, which is assumed to be known, and a feedback correction. Using the adaptive control algorithm to identify this correction, we demonstrated parameter and dynamic subsystem identification on a range of examples, including single and two-degree-of-freedom mass-spring-damper systems. Furthermore, we have presented results from an experimental setup, demonstrating the feasibility of applying these methods to real data and models from RLC circuits. Finally, we demonstrated the method on a space weather modeling problem, where the initial model is nonlinear and has no explicit representation.

\section{References} 1990.

${ }^{1}$ C. Minas and D. Inman, "Matching Finite Element Models to Modal Data," J. Vibration Acoust., Vol. 112, pp. 84-92,

${ }^{2}$ M. I. Friswell and J. E. Mottershead. Finite Element Model Updating in Structural Dynamics. Kluwer, Dordrecht, 1995.

${ }^{3}$ S. O. R. Moheimani, "Model Correction for Sampled-data Models of Structures," J. Guidance, Contr., Dynamics, Vol. 24, pp. 634-637, 2001.

${ }^{4}$ J. B. Carvalho, B. N. Datta, W. Lin, and C. Wang, "Symmetry Preserving Eigenvalue Embedding in Finite-element Model Updating of Vibrating Structures," J. Sound Vibration, Vol. 290, pp. 839-864, 2006.

${ }^{5}$ F. D'Andrea and R. Vautard, "Reducing Systematic Errors by Empirically Correcting Model Errors," Tellus, Vol. 52A, pp. $21-41,2000$.

${ }^{6}$ T. DelSole and A. Y. Hou, "Empirical Correction of a Dynamical Model. Part I: Fundamental Issues," Monthly Weather Rev., Vol. 127, pp. 2533-2545, 2001.

${ }^{7}$ C. M. Danforth, E. Kalnay, and T. Miyoshi, "Estimating and Correcting Global Weather Model Error," Monthly Weather Rev., Vol. 135, pp. 281-299, 2007.

${ }^{8}$ S. Mijanovic, G. E. Stewart, G. A. Dumont, and M. S. Davies, "A Controller Perturbation Technique for Transferring Closed-loop Stability between Systems," Automatica, Vol. 39, pp. 1783-1791, 2003.

${ }^{9}$ Z. Zhuquan, R. R. Bitmead, and M. Gevers, "H $\mathrm{H}_{2}$ Iterative Model Refinement and Control Robustness Enhancement," Proc. Conf. Dec. Contr., pp. 279-284, 1991.

${ }^{10}$ H. Palanthandalam-Madapusi, E. L. Renk, and D. S. Bernstein. Data-Based Model Refinement for Linear and Hammerstein Systems Using Subspace Identification and Adaptive Disturbance Rejection. Proc. Conf. Contr. Appl., pp. 1630-1635, Toronto, Canada, August 2005.

${ }^{11}$ M. A. Santillo, A. M. D'Amato, and D. S. Bernstein, "System Identification Using a Retrospective Correction Filter for Adaptive Feedback Model Updating," Proc. Amer. Contr. Conf., pp. 4392-4397, St. Louis, MO, June 2009.

${ }^{12}$ A. M. D'Amato and D. S. Bernstein, "Linear Fractional Transformation Identification Using Retrospective Cost Optimization," Proc. SYSID, pp. 450-455, Saint-Malo, France, July 2009.

${ }^{13}$ A. M. D'Amato, B. J. Arritt, J. A. Banik, E. V. Ardelean, and D. S. Bernstein, "Structural Health Determination and Model Refinement for a Deployable Composite Boom," AIAA SDM Conf., Palm Springs, CA, April 2009, AIAA-2009-2373.

${ }^{14}$ J.-N. Juang, Applied System Identification, Prentice Hall, 1993.

${ }^{15}$ L. Ljung, System Identification: Theory for the User, 2nd edition, Prentice Hall, 1999.

${ }^{16}$ P. Van Overschee and B. De Moor, Subspace Identification for Linear Systems: Theory, Implementation, Applications, Kluwer, 1996.

${ }^{17}$ J. S. Bendat, Nonlinear Systems Techniques and Applications, Wiley, 1989.

${ }^{18}$ J. Sjoberg et al., "Nonlinear Black-Box Modeling in System Identification: A Unified Overview," Automatica, Vol. 31, pp. 1691-1724, 1995.

${ }^{19}$ R. Haber and L. Keviczky, Nonlinear System Identification-Input-Output Modeling Approach Vol. 1: Nonlinear System Parameter Identification, Kluwer Academic Publishers, 1999.

${ }^{20}$ O. Nelles, Nonlinear System Identification, Springer, 2001.

${ }^{21}$ H. Palanthandalam-Madapusi, D. S. Bernstein, and A. J. Ridley, "Space Weather Forecasting: Identifying Periodically Switching Block-structured Models to Predict Magnetic-field Fluctuations," IEEE Contr. Sys. Mag., Vol. 27, pp. 109-123, October 2007.

${ }^{22}$ R. Venugopal and D. S. Bernstein. "Adaptive Disturbance Rejection Using ARMARKOV System Representations," IEEE Trans. Contr. Sys. Tech., Vol. 8, pp. 257-269, 2000.

${ }^{23}$ M. A. Santillo and D. S. Bernstein. A Retrospective Correction Filter for Discrete-time Adaptive Control of Nonminimum Phase Systems, in Proc. Conf. Dec. Contr., pp. 690-695, Cancun, Mexico, December 2008.

${ }^{24}$ J. B. Hoagg, M. A. Santillo, and D. S. Bernstein, "Discrete-Time Adaptive Command Following and Disturbance Rejection for Minimum Phase Systems with Unknown Exogenous Dynamics," IEEE Trans. Autom. Contr., Vol. 53, pp. 912-928, 2008.

${ }^{25}$ M. S. Holzel, M. A. Santillo, J. B. Hoagg, and D. S. Bernstein, "Adaptive Control of the NASA Generic Transport Model Using Retrospective Cost Optimization," Proc. AIAA Guid. Nav. Contr. Conf., Chicago, IL, August 2009, AIAA-2009-5616.

${ }^{26}$ M. A. Santillo and D. S. Bernstein, "Adaptive Control Based on Retrospective Cost Optimization," AIAA J. Guid. Contr. Dyn., Vol. 33, pp. 289-304, 2010.

${ }^{27}$ J. B. Hoagg and D. S. Bernstein, "Cumulative Retrospective Cost Adaptive Control with RLS-Based Optimization," Proc. Amer. Contr. Conf., Baltimore, MD, pp. 4016-4021, Baltimore, MD, June 2010. 
${ }^{28}$ A. M. D'Amato, K. S. Mitchell, A. R. Wu, S. L. Kukreja, and D. S. Bernstein, "Damage Localization for Structural Health Monitoring Using Retrospective Cost Model Refinement," Proc. 51th AIAA Structures, Dynamics, and Mechanics of Materials Conference, AIAA-2010-2628, Orlando, FL, April 2010.

${ }^{29}$ A. M. D'Amato, A. J. Ridley , and D. S. Bernstein, "Adaptive Model Refinement for the Ionosphere and Thermosphere," NASA CIDU, Mountain View, CA, October 2010.

${ }^{30}$ A. J. Ridley, Y. Deng, and G. T'oth. "The global ionosphere-thermosphere model". J. Atmos. Solar-Terr. Phys., 68(8), pp. 839-864, 2006. 